\title{
Skeletal Muscle Mass Change During Chemotherapy: A Systematic Review and Meta-analysis
}

\author{
MIN KYEONG JANG ${ }^{1,2}$, CHANG PARK ${ }^{2}$, SUSAN HONG ${ }^{1,3}$, HONGJIN LI $^{1,2}$, \\ ESTHER RHEE ${ }^{1,4}$ and ARDITH Z. DOORENBOS ${ }^{1,2}$ \\ ${ }^{1}$ University of Illinois Cancer Center, Chicago, IL, U.S.A.; \\ ${ }^{2}$ Department of Biobehavioral Health Science, \\ University of Illinois at Chicago, College of Nursing, Chicago, IL, U.S.A.; \\ ${ }^{3}$ Department of Medicine, Division of Academic Internal Medicine and Geriatrics, \\ University of Illinois at Chicago, College of Medicine, Chicago, IL, U.S.A.; \\ ${ }^{4}$ Department of Medicine, Division of Academic Internal Medicine, \\ University of Illinois at Chicago, College of Medicine, Chicago, IL, U.S.A.
}

\begin{abstract}
Background/Aim: Skeletal muscle mass loss is an emerging concern in oncology. Our systematic review and meta-analysis identified the mean difference in skeletal muscle index pre-to post-chemotherapy and synthesized potential key factors. Materials and Methods: We searched primary original research published through October 2019 in four databases: MEDLINE via PubMed, Scopus, CINAHL, and Embase. Results: Fifteen studies were included, $60 \%$ published in the past 2 years (2018-2019). Advanced non-small cell lung cancer was the most frequently reported cancer, and overall survival the most often identified key related factor. Mean difference in skeletal muscle index during chemotherapy was 2.72 (95\% CI=1.77-3.67, $p=0.00)$, with muscle loss in males $(4.52,95 \% C I=3.34-5.71, p=0.00)$ about 1.6 times higher than that in females $(2.86,95 \% \mathrm{CI}=0.81-4.92, p=0.01)$. Conclusion: Oncologists should recognize sex-specific differences in skeletal muscle mass loss during chemotherapy and consider adjusting treatment accordingly.
\end{abstract}

Low skeletal muscle mass is an emerging issue in oncology. While the amount of skeletal muscle mass loss varies widely across cancer types, between $5 \%$ and $89 \%$ of cancer patients

This article is freely accessible online.

Correspondence to: Min Kyeong Jang, Ph.D., University of Illinois Cancer Center, 818 S Wolcott Ave, Chicago, IL 60612, U.S.A. Tel: +1 3124134557, Fax: +1 3129968945, e-mail: mjang21@uic.edu

Key Words: Muscle mass loss, sarcopenia, cancer, skeletal muscle index, meta-analysis, review. have low skeletal muscle mass (1). Low skeletal muscle mass at baseline has been reported to the increase incidence of disability among cancer patients and is associated with poor anti-tumor response (2). In addition, cancer patients with low skeletal muscle mass during cancer treatment have been reported to have a higher risk of mortality (3-5), cancer recurrence (6-8), and reduced quality of life (9).

The causes of low skeletal muscle mass are multifactorial. These include cancer itself, cancer treatments, and aging (10). Chemotherapy is known to accelerate muscle mass loss in cancer patients. For example, gastric cancer patients who received adjuvant chemotherapy had significantly decreased skeletal muscle mass, which was an independent risk factor for overall survival rate (11). In addition, higher risk of chemotherapy toxicity has been related to low muscle mass, with $50 \%$ of metastatic breast cancer patients with sarcopenia, compared to $20 \%$ of those without sarcopenia, showing capecitabine toxicity (12). Since chemotherapy is linked to loss of muscle mass, which is in turn linked to an increased risk of mortality (12), cancer specialists need to recognize the importance of skeletal muscle mass as a predictor of survival rates.

Recent studies have offered reasons for viewing skeletal muscle mass change as an essential predictor of survival rates, regardless of whether a patient meets criteria for sarcopenia before or after treatment. In a previous retrospective study involving 394 patients with nasopharyngeal carcinomas, the presence of sarcopenia, whether before or after cancer treatment, was not related to overall survival. Severe muscle loss after chemotherapy, however, was an independent predictor of prognosis (13). In another retrospective study involving locally advanced cervical cancer patients, pretreatment sarcopenia was not related to overall survival. 
Records identified through database search $(n=1,048)$

PubMed ( $n=286)$, Scopus ( $n=285)$, CINAHL ( $n=85)$, Embase ( $n=392)$

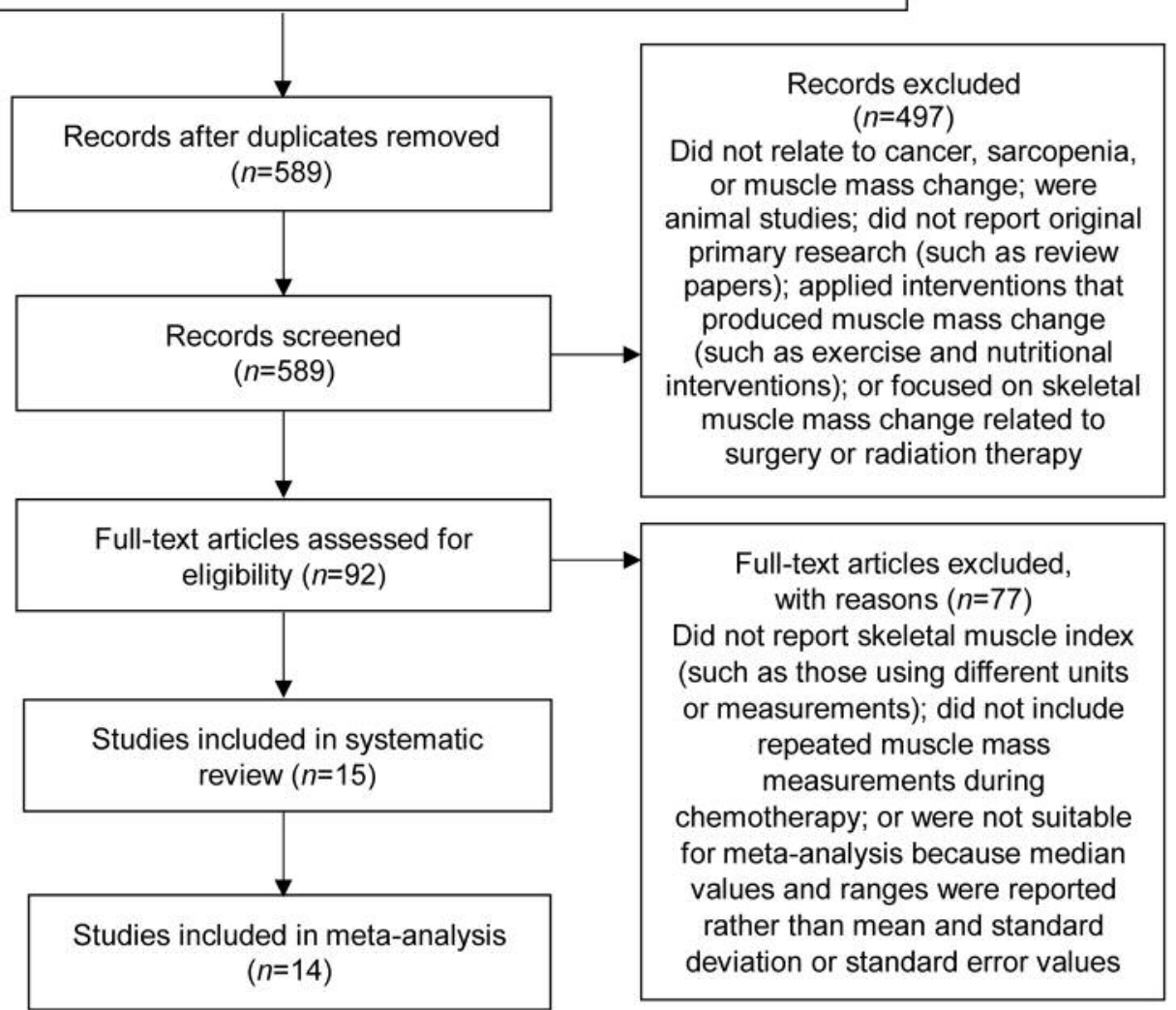

Figure 1. PRISMA flow diagram.

However, skeletal muscle mass loss during concurrent chemoradiation therapy was independently related to both poorer overall survival and cancer-specific survival (14). Past systematic review and meta-analysis papers have focused on low muscle mass either before treatment or after treatment. We found no such studies examining skeletal muscle mass change during chemotherapy. The focus of our meta-analysis thus reflects researchers' current areas of interest regarding skeletal muscle mass change and examines potential key factors related to skeletal muscle mass loss as well as mean differences in skeletal muscle mass change during treatment.

\section{Materials and Methods}

Data sources and search strategy. We performed a comprehensive search for relevant articles published from 1973 through October 2019 using four databases: MEDLINE via PubMed, Scopus, the Cumulative Index to Nursing and Allied Health Literature (CINAHL) Plus with Full Text, and the Excerpta Medica Database (Embase). Because the databases use differing Medical Subject Headings (MeSH) terms, a research librarian and expert researchers helped to identify MeSH terms likely to produce the most accurate search results. The general search terms were neoplasms (or cancer or oncology or cancer survivor) AND skeletal muscle index (or low muscle mass or sarcopenia or muscular atrophy) AND chemotherapy. Only English-language published articles for which full-text versions were available were included. Figure 1 illustrates the detailed search strategy employed. This systematic review and meta-analysis were guided by the Preferred Reporting Items for Systematic Reviews and Meta-Analyses (PRISMA) guidelines. Titles and abstracts were independently reviewed by two reviewers, and the full text of each relevant article was obtained. After selecting relevant studies, the two reviewers assessed their quality and extracted appropriate data.

Study selection. The following criteria were used to select studies for inclusion: (a) primary original research published in a journal; (b) a study sample consisting of cancer patients and survivors; (c) a study that included repeated measurements of skeletal muscle index using computed tomography (CT); and (d) a study that appeared in a published English-language article. We excluded studies that: (a) did not relate to cancer, sarcopenia, or muscle mass change; (b) were animal studies; (c) did not report original primary 
research (such as review papers); (d) did not report skeletal muscle index (such as those using different units or measurements); (e) applied interventions that produced muscle mass change (such as exercise and nutritional interventions); (f) did not include repeated muscle mass measurements during chemotherapy; ( $\mathrm{g}$ ) focused on skeletal muscle mass change related to surgery or radiation therapy; or (h) were not suitable for meta-analysis because median values and ranges were reported rather than mean and standard deviation (SD) or standard error (SE) values.

Data extraction and analysis. The data that were collected from each of the 15 included articles of the study sample included study characteristics, measurements of muscle mass (mean and SD, or mean and SE), mean time between CT assessments, and key findings associated with skeletal muscle mass. To assess the quality of the nonrandomized studies in this review, the Newcastle-Ottawa Scale was applied independently by two reviewers (15). Three categories, including selection of the study population, comparability, and description of the outcome, were assessed, and high-quality studies were defined as those meeting 7 or more out of 9 items.

To test for heterogeneity of the studies, we calculated $I^{2}$ statistics and Cochrane's Q statistics. The $I^{2}$ value is the ratio of the interstudy variance. If an $I^{2}$ value exceeded $50 \%$ and the $p$-value of $\chi^{2}$ was below 0.1 , we concluded that there was substantial heterogeneity according to the criteria suggested in the Cochrane Handbook for Systematic Reviews of interventions (16). To estimate mean differences for parallel group analysis, the skeletal muscle mass change values reported across the studies were used. Specifically, mean differences between skeletal muscle index $\left(\mathrm{cm}^{2} / \mathrm{m}^{2}\right)$ values measured pre- and post-chemotherapy were employed. To obtain the SE of the mean differences, the authors adhered to the following Cochrane Handbook guideline (16): $\mathrm{SE}=\mathrm{SD}$ of within-participant differences between pre- and postchemotherapy measurements divided by the square root of the number of participants. Effect sizes were derived using both the mean skeletal muscle index differences (pre- and postchemotherapy) and the SE. Subsequently, the authors performed a meta-analysis using a random-effects model to investigate skeletal muscle index change. Between-study heterogeneity was assessed by visually inspecting forest plots generated from the study data. To identify sex-specific differences in skeletal muscle mass changes, we applied subgroup meta-analysis to explore the heterogeneity of the studies.

\section{Results}

Study characteristics and systematic review. A total of 1,048 articles, including duplicate entries in the databases, were identified as potentially relevant based on the final search terms employed. After the initial screening of titles and abstracts, we selected 15 articles for detailed analysis (Figure $1)$. With respect to quality assessment, all 15 of the studies reviewed were rated as high quality; their mean rating of quality assessment was 8.37, with a range from 6 to 9 .

Table I shows study characteristics and the number of studies that found these characteristics to be significantly related to skeletal muscle mass loss. Table II provides more detailed information for each study reviewed. The 15
Table I. General analysis of the studies reviewed $(N=15)$.

\begin{tabular}{llr}
\hline Category & Description & $\mathrm{N}$ \\
\hline Year of publication & 2015 & 3 \\
& 2016 & 1 \\
& 2017 & 2 \\
& 2018 & 3 \\
& 2019 & 6
\end{tabular}

Level of evidence $\quad$ Level IV: Case-control or cohort study 15

$\begin{array}{lr}\text { Nations } & \text { Japan } \\ \text { China } & 3 \\ \text { Korea } & 2 \\ \text { Ireland } & 2 \\ \text { The Netherlands } & 1 \\ \text { Taiwan } & 1 \\ \text { United Kingdom } & 1 \\ \text { United States } & 1\end{array}$

Cancer type (site) Advanced non-small cell lung cancer 3

Esophageal cancer 2

Gastric cancer $\quad 2$

Pancreatic cancer $\quad 1$

Head and neck squamous cell carcinoma 1

Locally advanced cervical cancer $\quad 1$

Advanced endometrial cancer $\quad 1$

Nasopharyngeal carcinoma 1

Colorectal liver metastases $\quad 1$

Unresectable pancreatic

cancer or distal cholangiocarcinoma

Advanced biliary tract cancer

Factors contributing to skeletal muscle

Overall survival

Gender

mass loss

Mortality risk

Acute toxicities

Adjuvant chemotherapy

Body weight

Cancer-specific survival

Carcinoembryonic antigen

$\geq 200 \mathrm{ng} / \mathrm{ml}$ (preoperative)

Decreased body mass index

during chemotherapy

Disease-free survival

Extended-field radiotherapy

Hand-grip strength

Incremental shuttle walking distance

Primary tumor origin 1

Performance status 1

Recurrence-free survival 1

Smoker

Therapy type (cytotoxic chemotherapy 1

versus molecular targeted therapy)

Tumor type

Tumor progression

Tumor diameter change

Tumor stage

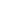

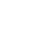

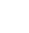

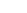

(1)

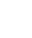

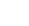


Table II. Summary of the 15 articles reviewed.

\begin{tabular}{|c|c|c|c|c|c|c|c|}
\hline $\begin{array}{l}\text { Author (year) } \\
\text { Country } \\
\text { (Citation) }\end{array}$ & $\begin{array}{c}\text { Sample } \\
\text { T/M/F }\end{array}$ & $\begin{array}{l}\text { Mean age, } y r \\
( \pm \text { SD) or } \\
\text { (range) }\end{array}$ & $\begin{array}{l}\text { Study design/ } \\
\text { mean time between } \\
\text { assessments }\end{array}$ & Cancer type & Cancer treatment & $\begin{array}{c}\text { Mean SMI at } \\
\text { diagnosis, } \\
\mathrm{cm}^{2} / \mathrm{m}^{2} \\
( \pm \mathrm{SD} \text { or } \mathrm{SE})\end{array}$ & $\begin{array}{c}\text { Mean SMI after } \\
\text { chemotherapy, } \\
\mathrm{cm}^{2} / \mathrm{m}^{2} \\
( \pm \mathrm{SD} \text { or } \mathrm{SE})\end{array}$ \\
\hline
\end{tabular}

\begin{tabular}{lccc}
\hline $\begin{array}{l}\text { Cho } \text { et al. } \\
(2017)\end{array}$ & $524 /$ & T: $61( \pm 9.4)$ & Retrospective/ \\
$\begin{array}{l}\text { Korea } \\
(35)\end{array}$ & $344 /$ & M: $61( \pm 9.5)$ & $\begin{array}{c}\text { no specific time } \\
\text { data provided }\end{array}$ \\
$\begin{array}{l}\text { Griffin } \text { et al. } \\
(2019)\end{array}$ & $78 /$ & T: $61( \pm 9.2)$ & \\
$\begin{array}{l}\text { Ireland } \\
(12)\end{array}$ & $37 /$ & & $\begin{array}{c}\text { Retrospective/ } \\
182 \text { days }\end{array}$ \\
& 41 & & \\
(range=72-316)
\end{tabular}

$\begin{array}{lc}\text { Guinan et al. } & 28 / \\ \text { (2018) } & 23 / \\ \text { Ireland } & 5\end{array}$

(30)

$\begin{array}{lc}\begin{array}{l}\text { Huang } \text { et al. } \\ (2019)\end{array} & 394 / \\ \text { China } & 298 / \\ (13) & 96 \\ & \\ & \\ \text { Jung } \text { et al. } & 258 / \\ (2019) & 223 / \\ \text { Korea } & 35 \\ (36) & \\ \text { Kakinuma } \text { et al. } & 65 / \\ \text { (2018) } & 40 / \\ \text { Japan } & 25 \\ \text { (37) } & \end{array}$

(37)

$\begin{array}{lccc}\text { Kimura } \text { et al. } & 134 / & \text { M: } 66(36-86) & \text { Retrospective/ } \\ \begin{array}{l}\text { (2015) } \\ \text { Japan }\end{array} & 80 / & \text { F: } 66(35-81) & 29.3 \text { months } \\ \text { (38) } & 54 & & \text { (range: } 26.3-34.2)\end{array}$

(38)

$\begin{array}{lcc}\text { Lee } \text { et al. } & 245 / & \text { T: } 63( \pm 12.7) \\ (2018) & 0 / & \\ \text { Taiwan } & 245 & \\ \begin{array}{l}(14) \\ \text { Lee } \text { et al. }\end{array} & 131 / & \text { T: } 54( \pm 9.6) \\ (2019) & 0 / & \\ \text { China } & 131 & \\ (39) & & \end{array}$

\section{Prospective observational design/92 days (range: 61-118)}

T: 46 (18-79)

T: 64 (56-73)

Prospective/

53.6 months (range: 26.3-70.5)

T: $66( \pm 10.5)$
Retrospective/ 132 days
22.7 months
Retrospective (range: 2.5-46.4)

Retrospective/ 4.8 months (range: 3.5-5.9)
Retrospective/ 223 days (range: 205-236)

Nasopharyngeal
carcinoma

Esophageal
cancer

Advanced
biliary
tract cancer
Pancreatic
cancer

39.9\%: gemcitabine/ platinum $37.6 \%$ :

5-FU/platinum

44\%: FOLFIRINOX

19\%: gemcitabine+ nab-paclitaxel

11\%: gemcitabine (single agent)

13\%: gemcitabine+ oxaliplatin

4\%: gemcitabine+ cis/carboplatin 5\%: 5 -FU

79\%: CROSS

(cisplatin/5-FU,

$40 \mathrm{~Gy} / 15 \mathrm{fr}$, or

carboplatin/paclitaxel,

$41.4 \mathrm{~Gy} / 23 \mathrm{fr}$ )

21\%: MAGIC

(etoposide, cisplatin,

FU, or capecitabine) $11 \%$ : radical

radiotherapy $\pm \mathrm{TT}$

43\%: CCRT $\pm \mathrm{TT} / \mathrm{AC}$

45\%: IC + CCRT

$$
\pm \mathrm{TT} / \mathrm{AC}
$$

$13 \%:$ IC + radical radiotherapy

Head and neck squamous cell carcinoma
Advanced non- small cell lung cancer

$12 \%$ : surgery + CRT $36 \%$ : CRT

$66 \%$ : platinum-based combination $2 \%$ : single agent (cytotoxic drug) $32 \%$ : single agent (EGFR-TKI or ALK-TKI)

Advanced non- $68.8 \%(\mathrm{M}), 59.3 \%(\mathrm{~F})$ : platinum-based combination lung cancer

$18.8 \%(\mathrm{M}), 5.6 \%(\mathrm{~F})$ : single-agent (cytotoxic drug) $12.5 \%(\mathrm{M}), 35.2 \%(\mathrm{~F})$ : single-agent (EGFR-TKI)

86.5\%: chemotherapy

advanced cervical cancer

$$
\begin{array}{cc}
\text { Advanced } & 97 \%: 6 \text { cycles of } \\
\text { endometrial } & \text { paclitaxel and } \\
\text { cancer } & \text { carboplatin AUC5 }
\end{array}
$$

T: $46.02( \pm 8.38) \quad$ T: $40.67( \pm 10.40)$

M: $48.58( \pm 7.74) \quad$ M: $42.86( \pm 9.52)$

F: $41.24( \pm 7.39) \quad$ F: $36.63( \pm 12.02)$

T: $45.6( \pm 8.7)$

T: $42.3( \pm 9.3)$

T: $60.3( \pm 8.1)$

T: $54.7( \pm 7.5)$

$\mathrm{T}: 42.8( \pm 8.4)$

M: $45.4( \pm 7.5)$

F: $34.7( \pm 5.5)$

T: $38.1( \pm 8.0)$

M: $40.4( \pm 6.8)$

F: $30.9( \pm 7.0)$

T: $61.7( \pm 8.4)$

T: $57.9( \pm 4.7)$

T: $44.0( \pm 0.9)$

T: $40.7( \pm 0.8)$

M: $46.4( \pm 6.8)$

M: $43.46( \pm 0.55)$

F: $37.0( \pm 4.5) \quad$ F: $36.98( \pm 0.50)$

T: $39.6( \pm 7.6)$

T: $39.3( \pm 7.7)$
T: $42.4( \pm 6.5)$

T: $42.3( \pm 7.6)$ 
Table II. Continued

\begin{tabular}{|c|c|c|c|c|c|c|c|}
\hline $\begin{array}{l}\text { Author (year) } \\
\text { Country } \\
\text { (Citation) }\end{array}$ & $\begin{array}{l}\text { Sample } \\
\text { T/M/F }\end{array}$ & $\begin{array}{l}\text { Mean age, yr } \\
( \pm \mathrm{SD}) \text { or } \\
(\text { range })\end{array}$ & $\begin{array}{c}\text { Study design/ } \\
\text { mean time between } \\
\text { assessments }\end{array}$ & Cancer type & Cancer treatment & $\begin{array}{l}\text { Mean SMI at } \\
\text { diagnosis, } \\
\mathrm{cm}^{2} / \mathrm{m}^{2} \\
( \pm \mathrm{SD} \text { or } \mathrm{SE})\end{array}$ & $\begin{array}{c}\text { Mean SMI after } \\
\text { chemotherapy, } \\
\mathrm{cm}^{2} / \mathrm{m}^{2} \\
( \pm \mathrm{SD} \text { or SE })\end{array}$ \\
\hline $\begin{array}{l}\text { Li et al. } \\
(2019) \\
\text { China } \\
(40)\end{array}$ & $\begin{array}{c}153 / \\
101 / \\
52\end{array}$ & T: $52(26-80)$ & $\begin{array}{l}\text { Retrospective/ } \\
36 \text { months } \\
\text { (range:7-115) }\end{array}$ & Gastric cancer & $\begin{array}{c}\text { Adjuvant } \\
\text { chemoradiotherapy } \\
\text { (5-FU and leucovorin) }\end{array}$ & T: $39.8( \pm 11.1)$ & T: $38.2( \pm 9.3)$ \\
\hline $\begin{array}{l}\text { Naito et al. } \\
\text { (2017) } \\
\text { Japan } \\
(41)\end{array}$ & $\begin{array}{l}30 / \\
19 / \\
11\end{array}$ & $\begin{array}{l}\text { T: } 74(70-82) \\
\text { M: } 74(70-82) \\
\text { F: } 76(70-80)\end{array}$ & $\begin{array}{l}\text { Prospective/ } \\
10 \text { months }\end{array}$ & $\begin{array}{l}\text { Advanced non- } \\
\text { small cell } \\
\text { lung cancer }\end{array}$ & $\begin{array}{l}80 \% \text { : cytotoxic } \\
\text { regimen } \\
20 \%: \text { targeted } \\
\text { regimen }\end{array}$ & $\begin{array}{c}\text { T: } 41.2( \pm 7.8) \\
\text { M: } 44.5( \pm 7.6) \\
\text { F: } 35.4( \pm 4.1)\end{array}$ & T: $39.4( \pm 0.7)$ \\
\hline $\begin{array}{l}\text { Yamaoka et al. } \\
\text { (2015) } \\
\text { Japan } \\
(11)\end{array}$ & $\begin{array}{c}102 / \\
71 / \\
31\end{array}$ & T: $64( \pm 10.5)$ & $\begin{array}{c}\text { Retrospective/ } \\
1 \text { year (range: } \\
0.90-1.10)\end{array}$ & Gastric cancer & $\begin{array}{l}\text { 63\%: adjuvant } \\
\text { chemotherapy } \\
\text { (none or within } \\
6 \text { months) } \\
37 \% \text { : adjuvant } \\
\text { chemotherapy } \\
\text { (after } 6 \text { months) }\end{array}$ & $\mathrm{T}: 46.6( \pm 7.84)$ & $\mathrm{T}: 43.6( \pm 6.92)$ \\
\hline $\begin{array}{l}\text { Okuno et al. } \\
\text { (2019) } \\
\text { USA } \\
(42)\end{array}$ & $\begin{array}{c}169 / \\
97 / \\
72\end{array}$ & $\mathrm{~T}: 56( \pm 11.7)$ & $\begin{array}{l}\text { Retrospective/ } \\
47 \text { months }\end{array}$ & $\begin{array}{l}\text { Colorectal liver } \\
\text { metastases }\end{array}$ & $\begin{array}{l}\text { 89\%: oxaliplatin } \\
19 \% \text { : irinotecan } \\
12 \% \text { : multiple } \\
\text { regimens } \\
78 \% \text { : bevacizumab } \\
\text { 10\%: cetuximab/ } \\
\text { panitumumab }\end{array}$ & T: $51.2( \pm 10.6)$ & T: $50.6( \pm 10.7)$ \\
\hline $\begin{array}{l}\text { Reisinger } \text { et al. } \\
\text { (2015) } \\
\text { the Netherlands }\end{array}$ & $\begin{array}{l}123 / \\
(43) \\
101 / \\
22\end{array}$ & $\mathrm{~T}: 63( \pm 10)$ & $\begin{array}{l}\text { Prospective/ } \\
111 \text { days (range: } \\
94-128 \text { ) }\end{array}$ & $\begin{array}{l}\text { Esophageal } \\
\text { cancer }\end{array}$ & $\begin{array}{l}\text { 93\%: neoadjuvant } \\
\text { CRT (cisplatin/ } \\
\text { 5-FU or paclitaxel/ } \\
\text { carboplatin or } \\
\text { epirubicin/cisplatin/ } \\
\text { capecitabine) }\end{array}$ & $\begin{array}{c}\text { T: } 50.9( \pm 8.5) \\
\text { M: } 53.4( \pm 7.8) \\
\text { F: } 42.7( \pm 5.4)\end{array}$ & $\begin{array}{c}\text { T: } 48.4( \pm 8.5) \\
\text { M: } 49.5( \pm 7.9) \\
\text { F: } 39.7( \pm 4.4)\end{array}$ \\
\hline $\begin{array}{l}\text { Rollins et al. } \\
(2016) \\
\text { UK } \\
(44)\end{array}$ & $\begin{array}{l}228 / \\
123 / \\
105\end{array}$ & $\mathrm{~T}: 69( \pm 10.9)$ & $\begin{array}{l}\text { Retrospective/ } \\
60 \text { days }\end{array}$ & $\begin{array}{c}\text { Pancreatic cancer } \\
\text { or distal } \\
\text { cholangio- } \\
\text { carcinoma }\end{array}$ & $\begin{array}{c}\text { Palliative } \\
\text { chemotherapy }\end{array}$ & $\mathrm{T}: 42.4( \pm 8.6)$ & T: $39.8( \pm 8.0)$ \\
\hline
\end{tabular}

$\mathrm{N}=15$ (number of studies). Data are reported for study totals (T) and for male (M) and female (F) where available. ALK-TKI: Anaplastic lymphoma kinase tyrosine kinase inhibitor; AUC: area under the curve; CCRT: concurrent chemoradiotherapy; CRT: chemoradiotherapy; EGFR-TKI: epidermal growth factor receptor tyrosine kinase inhibitor; FU: fluorouracil; IC: induction chemotherapy; MAGIC: etoposide, cisplatin, fluorouracil or capecitabine; SMI: skeletal muscle index; TT: targeted therapy; TT/AC: targeted therapy/adjuvant chemotherapy.

articles involved only case-control or cohort (level IV) study designs (17). Across the 15 articles, the average age of participants was $60 \pm 6.5$ years. A total of 2,662 participants produced the results synthesized herein, and the mean sample size was $177 \pm 136.4$, with a range from 28 to 524 . Notably, $60 \%$ of the articles were published in the past 2 years (2018-2019). A large variety of cancer types were addressed in these studies; advanced non-small cell lung cancer was the most frequently reported, followed by esophageal cancer and gastric cancer equally. Among the various factors related to skeletal muscle mass change, overall survival was the factor most often identified, followed by mortality risk and sex.
Meta-analysis. Meta-analysis of 14 studies was performed for skeletal muscle mass change outcomes. One study did not report total skeletal muscle index values, instead it reported separate male and female skeletal muscle index results; consequently, although this study was included in the systematic review, it was not subjected to meta-analysis. Forest plots were constructed for each outcome using the mean effect size measured by the random-effects model (Figure 2).

Statistically significant heterogeneity was found $\left(I^{2}=86.83 \%\right.$, $\mathrm{Q}=132.20, p=0.00$ ), and thus we employed a random-effects method. The summary mean difference, which was derived from the 14 studies with a total of 2,528 participants, revealed 


\begin{tabular}{|c|c|c|c|}
\hline Study & & $\begin{array}{c}\text { Effect Size } \\
\text { with } 95 \% \mathrm{Cl}\end{array}$ & $\begin{array}{c}\text { Weight } \\
(\%)\end{array}$ \\
\hline Cho et al., 2017 & & $5.35[4.63,6.07]$ & 8.43 \\
\hline Griffin et al., 2019 & & $3.30[1.37,5.23]$ & 6.48 \\
\hline Guinan et al., 2018 & & $5.60[2.60,8.60]$ & 4.70 \\
\hline Huang et al., 2019 & & $4.70[3.87,5.53]$ & 8.30 \\
\hline Jung et al., 2019 & & $3.80[2.78,4.82]$ & 8.03 \\
\hline Kakinuma et al., 2018 & & $3.30[1.54,5.06]$ & 6.78 \\
\hline Lee et al., 2018 & & $0.30[-0.65,1.25]$ & 8.13 \\
\hline Lee et al., 2019 & & $0.10[-1.01,1.21]$ & 7.90 \\
\hline Li et al., 2019 & & $1.60[-0.16,3.36]$ & 6.79 \\
\hline Naito et al., 2017 & & $1.80[-0.99,4.59]$ & 5.02 \\
\hline Yamaoka et al., 2015 & & $3.00[1.48,4.52]$ & 7.22 \\
\hline Okuno et al., 2019 & & $0.60[-1.00,2.20]$ & 7.08 \\
\hline Reisinger et al., 2015 & & $2.50[1.00,4.00]$ & 7.25 \\
\hline Rollins et al., 2016 & & $2.60[1.48,3.72]$ & 7.89 \\
\hline Overall & & $2.72[1.77,3.67]$ & \\
\hline \multicolumn{4}{|l|}{ Heterogeneity: $\mathrm{T}^{2}=2.65, \mathrm{I}^{2}=86.83 \%, \mathrm{H}^{2}=7.59$} \\
\hline \multicolumn{4}{|l|}{ Test of $\theta_{i}=\theta_{j}: Q(13)=132.20, p=0.00$} \\
\hline \multicolumn{4}{|l|}{ Test of $\theta=0: z=5.62, p=0.00$} \\
\hline & 10 & & \\
\hline
\end{tabular}

Figure 2. Random-effects meta-analysis results for skeletal muscle mass change in patients with cancer.

a significant loss of skeletal muscle mass (mean difference in skeletal muscle index $=2.72,95 \% \mathrm{CI}=1.77-3.67, p=0.00$ ).

In addition, four studies reported skeletal muscle index according to sex (Figure 3). Based on the mean difference by sex, statistically significant heterogeneity was found in male participants $\left(I^{2}=78.21 \%, \mathrm{Q}=12.22, p=0.01\right)$. Skeletal muscle mass loss was found in male participants (mean difference in skeletal muscle index $=4.52,95 \% \mathrm{CI}=3.34-5.71$, $p=0.00)$. In female participants, statistically significant heterogeneity was also found in the effect size $\left(I^{2}=89.98 \%\right.$, $\mathrm{Q}=34.25, p=0.00)$. In addition, loss of skeletal muscle mass was found (mean difference in skeletal muscle index $=2.86$, $95 \% \mathrm{CI}=0.81-4.92, p=0.01)$. Notably, skeletal muscle mass loss in males was about 1.6 times higher than that found in females.

\section{Discussion}

This systematic review and meta-analysis contribute to our understanding of the current research status on changes in skeletal muscle mass during cancer treatment. Although sarcopenia has been recognized as an important issue in oncology over the past 10 years, it is only recently that studies have focused on comparing skeletal muscle index before and after cancer treatment. Skeletal muscle mass change during treatment is recognized as a predictor of chemotherapy toxicity as well as of overall survival rates. Thus, this study's contributions include identification of key factors related to muscle mass loss, quantification of muscle mass change in terms of mean differences, and identification of directions for future study.

Among the findings of this systematic review, it is interesting to note that all 15 included studies focused on skeletal muscle mass comparison during treatment were published since 2015 , and $60 \%$ of them were published in the past 2 years (2018-2019). This finding indicates that crosssectional measurements of skeletal muscle index are trending toward longitudinal measurements in order to identify patterns of skeletal muscle mass change. Consequently, it appears that oncologists and researchers are coming to recognize the clinical significance of patterns of skeletal muscle mass change, especially with regard to patient survival.

In addition, although other review articles have reported studies providing sarcopenia findings (18-20), comparative studies of skeletal muscle mass change during chemotherapy that involve skeletal muscle index measurements have been scarce. In fact, although loss of skeletal muscle mass is a measurable predictor of cancer treatment toxicity, skeletal 
Male-specific studies

\begin{tabular}{l} 
Study \\
\hline Cho et al., 2017 \\
Huang et al., 2019 \\
Kimura et al., 2015 \\
Reisinger et al., 2015 \\
Overall \\
Heterogeneity: $\mathrm{T}^{2}=1.10, \mathrm{I}^{2}=78.21 \%, \mathrm{H}^{2}=4.59$ \\
Test of $\theta_{1}=\theta_{j}: \mathrm{Q}(3)=12.22, p=0.01$ \\
Test of $\theta=0: \mathrm{z}=7.50, p=0.00$
\end{tabular}

Female-specific studies

\begin{tabular}{l} 
Study \\
\hline Cho et al., 2017 \\
Huang et al., 2019 \\
Kimura et al., 2015 \\
Reisinger et al., 2015 \\
Overall \\
Heterogeneity: $\mathrm{T}^{2}=3.85, \mathrm{I}^{2}=89.98 \%, \mathrm{H}^{2}=9.98$ \\
Test of $\theta_{\mathrm{i}}=\theta_{\mathrm{j}}: \mathrm{Q}(3)=34.25, p=0.00$ \\
Test of $\theta=0: \mathrm{z}=2.73, p=0.01$
\end{tabular}

Figure 3. Random-effects meta-analysis results for skeletal muscle mass change in patients with cancer, by sex.

muscle mass change during treatment has not been widely studied by race/ethnicity or by country; this is true despite the fact that the incidence of sarcopenia is known to differ by race/ethnicity (21-24). Clearly more research is needed, particularly in Western countries, to better understand the relationships of race/ethnicity and culture to skeletal muscle mass change.

Of the various cancer types addressed, advanced nonsmall cell lung cancer was the most frequently reported, followed by gastric cancer and esophageal cancer equally. In the studies involving advanced non-small cell lung cancer, changes in skeletal muscle index varied, ranging from 0.2 to 3.3. Similarly, a previous systematic review and metaanalysis revealed that in one study, non-small cell lung cancer showed the highest prevalence of sarcopenia at baseline (74\%) (20). In addition, the study concluded that maintaining and gaining skeletal muscle cross-sectional area was significantly related to longer overall patient survival (25). In another review paper, focused on gastric cancer, (26), the authors pointed out that among patients with gastric cancer associated with eating disorders, loss of weight and muscle was often experienced, highlighting the importance of sarcopenia. Considering that chemotherapy regimens and cancer-related symptoms differ by cancer type, future researchers should compare skeletal muscle mass change among various cancer types, explore which regimens and cancer types result in the greatest skeletal muscle index loss, and identify predictors of skeletal muscle index change. These efforts may generate information that can be used to optimize interventions to better preserve and improve skeletal muscle mass.

In our systematic review, key factors related to skeletal muscle mass change included clinical, demographic, physical, and health-related factors. To be specific, overall survival was most often reported as a key factor, followed by mortality risk and sex. In addition, skeletal muscle mass loss showed associations with higher tumor stage, acute toxicities, lower physical function (in terms of hand-grip strength), chemotherapy regimens, cancer treatment type, tumor type, and tumor diameter change. While we identified 
a considerable variety of key factors related to skeletal muscle mass loss, we noted that cancer-related symptoms such as fatigue, depression, pain, and sleep disorder have seldom been considered in terms of their relationship with skeletal muscle mass loss. Considering that most cancer patients experience a variety of symptoms (27-29), studies examining cancer-related symptoms and associated skeletal muscle mass change should be conducted.

In the meta-analysis portion of our study, 14 studies reporting skeletal muscle mass change during cancer treatment were analyzed. No randomized controlled trials were included in this review, in order to avoid confounding factors (such as exercise and nutrition interventions) that affect muscle mass change. In our most significant finding, the mean value for skeletal muscle mass loss was 2.72 (95\% CI $\left.=1.77-3.67, \quad p=0.00, \quad I^{2}=86.83 \%\right)$; the high $I^{2}$ indicates that significant heterogeneity was observed across the 14 studies. In addition, although advanced non-small cell lung cancer was the most common cancer type observed in the systematic review, the meta-analysis revealed that patients with esophageal cancer (30) experienced the greatest skeletal muscle mass loss (skeletal muscle index change: 5.6, ranging from 3.7 to 7.5 ) between pre- and post-neoadjuvant therapy (mean time interval: 92 days, ranging from 61 to 118 days). In addition, $89 \%$ of those cancer patients used the CROSS protocol (cisplatin/5-fluorouracil, $40 \mathrm{~Gy} / 15 \mathrm{fr}$, or carboplatin/paclitaxel, $41.4 \mathrm{~Gy} / 23 \mathrm{fr}$ ) adapted from the Dutch ChemoRadiotherapy for Oesophageal cancer followed by Surgery Study (CROSS) trial. In a previous retrospective study involving patients with esophageal cancer who underwent neoadjuvant chemoradiotherapy, patients with sarcopenia were more likely to experience severe adverse events such as fever, mucositis, and neutropenic fewer than patients without sarcopenia (31). Thus, while patients with multiple cancer types exhibited significant skeletal muscle mass loss during treatment, esophageal cancer patients undergoing neoadjuvant therapy showed twice as much skeletal muscle mass loss compared to other cancers. This may be due to the fact that eating is more challenging for esophageal cancer patients.

Among the 14 studies, 4 showed specific skeletal muscle mass change by sex. Notably, the skeletal muscle mass loss in males was about 1.6 times higher than in females. In a previous study on risk factor analysis for sarcopenia among cancer patients, males were more likely to develop sarcopenia than females (32). Possible explanations for the differences in skeletal muscle mass loss by sex include hormonal, inflammatory, and myocellular mechanisms that affect underlying biological processes that promote fat deposition and loss of lean mass and strength (33). Sexspecific hormone changes due to testosterone may be one factor that affects muscle and fat composition (34). Another possibility may be that since the male skeletal muscle index at baseline is higher than that of females (4.54 vs. 2.86), males may be more susceptible to chemotherapy's effects on skeletal muscle mass loss. Also, it is necessary to take a closer look at adherence to health-related behavioral interventions such as nutrition and exercise during cancer treatment by sex. Since only 4 of the 14 studies compared skeletal muscle index by sex, future studies examining differences in skeletal muscle index by sex during chemotherapy are needed.

This meta-analysis has some limitations that should be acknowledged. To accurately compare skeletal muscle indexes and avoid confounding factors, this meta-analysis did not include intervention studies that involved maintaining or increasing skeletal muscle index. Future systematic reviews and meta-analyses should focus on reports of nutrition and exercise interventions to better understand their effectiveness at preserving skeletal muscle mass during chemotherapy. In addition, future studies should pay attention to a number of other factors potentially contributing to skeletal muscle mass loss, including social determinants of health, recurrence status, pre-existing medical conditions, and other cancer treatments received (such as surgery, radiation therapy, hormonal therapy, or immunotherapy). Finally, more than three-quarters of the studies identified for this meta-analysis employed retrospective designs; additional prospective studies are needed to more accurately determine risk factors for skeletal muscle mass loss while also avoiding confounding factors.

In summary, the findings derived from 14 studies revealed that total skeletal muscle mass significantly declined during chemotherapy and that male patients experienced a relative muscle mass loss 1.6 times greater than female patients. Also, esophageal cancer patients undergoing chemotherapy were found to be at serious risk for skeletal muscle mass loss. Therefore, health care providers should recognize sexspecific differences in muscle mass loss and consider adjusting patients' treatment regimens accordingly.

\section{Conflicts of Interest}

The Authors have no conflicts of interest concerning this study.

\section{Authors' Contributions}

M.K.J. designed the aim of the review, wrote the article, and supervised all steps in producing the article. C.P. contributed to the process and findings of the meta-analysis. S.H., H.L., E.R., and A.Z.D. contributed equally to this work, generated the figures, and wrote the article.

\section{Acknowledgements}

The Authors gratefully acknowledge the assistance of Rebecca Raszewski, MS, AHIP, with the database search performed for this study. This work was supported by the National Institute of Nursing 
Research of the US National Institutes of Health (K24NR015340) The content is solely the responsibility of the Authors and does not necessarily represent the official views of the National Institutes of Health.

\section{References}

1 Rier HN, Jager A, Sleijfer S, Maier AB and Levin MD: The prevalence and prognostic value of low muscle mass in cancer patients: a review of the literature. Oncologist 21: 1396-1409, 2016. PMID: 27412391. DOI: 10.1634/theoncologist.2016-0066

2 Davis MP and Panikkar R: Sarcopenia associated with chemotherapy and targeted agents for cancer therapy. Ann Palliat Med 8: 86-101, 2018. PMID: 30525762. DOI: 10.21037/apm. 2018.08.02

3 Psutka SP, Carrasco A, Schmit GD, Moynagh MR, Boorjian SA, Frank I, Stewart SB, Thapa P, Tarrell RF and Cheville JC: Sarcopenia in patients with bladder cancer undergoing radical cystectomy: impact on cancer-specific and all-cause mortality. Cancer 120: 2910-2918, 2014. PMID: 24840856. DOI: $10.1002 /$ cncr.28798

4 Huang D, Chen X, Chen X, Wang S, Shen X, Chen X, Yu Z and Zhuang C: Sarcopenia predicts 1-year mortality in elderly patients undergoing curative gastrectomy for gastric cancer: a prospective study. J Cancer Res Clin Oncol 142: 2347-2356, 2016. PMID: 27573385. DOI: 10.1007/s00432-016-2230-4

5 Reisinger KW, Bosmans JW, Uittenbogaart M, Alsoumali A, Poeze M, Sosef MN and Derikx JP: Loss of skeletal muscle mass during neoadjuvant chemoradiotherapy predicts postoperative mortality in esophageal cancer surgery. Ann Surg Oncol 22: 44454452, 2015. PMID: 25893413. DOI: 10.1245/s10434-015-4558-4

6 Prado CM, Baracos VE, McCargar LJ, Reiman T, Mourtzakis M, Tonkin K, Mackey JR, Koski S, Pituskin E and Sawyer MB: Sarcopenia as a determinant of chemotherapy toxicity and time to tumor progression in metastatic breast cancer patients receiving capecitabine treatment. Clin Cancer Res 15: 2920-2926, 2009. PMID: 19351764. DOI: 10.1158/1078-0432.CCR-08-2242

7 Chang KV, Chen JD, Wu WT, Huang KC, Hsu CT and Han DS: Association between loss of skeletal muscle mass and mortality and tumor recurrence in hepatocellular carcinoma: a systematic review and meta-analysis. Liver Cancer 7: 90-103, 2018. PMID: 29662836. DOI: 10.1159/000484950

8 Kamachi S, Mizuta T, Otsuka T, Nakashita S, Ide Y, Miyoshi A, Kitahara K, Eguchi Y, Ozaki I and Anzai K: Sarcopenia is a risk factor for the recurrence of hepatocellular carcinoma after curative treatment. Hepatol Res 46: 201-208, 2016. PMID: 26223826. DOI: 10.1111/hepr.12562

9 Nipp RD, Fuchs G, El-Jawahri A, Mario J, Troschel FM, Greer JA, Gallagher ER, Jackson VA, Kambadakone A, Hong TS, Temel JS and Fintelmann FJ: Sarcopenia Is associated with quality of life and depression in patients with advanced cancer. Oncologist 23: 97-104, 2018. PMID: 28935775. DOI 10.1634/theoncologist.2017-0255

10 Williams GR, Rier HN, McDonald A and Shachar SS: Sarcopenia \& aging in cancer. J Geriatr Oncol 10: 374-377, 2019. PMID: 30343999. DOI: 10.1016/j.jgo.2018.10.009

11 Yamaoka Y, Fujitani K, Tsujinaka T, Yamamoto K, Hirao M and Sekimoto M: Skeletal muscle loss after total gastrectomy, exacerbated by adjuvant chemotherapy. Gastric Cancer 18: 382389, 2015. PMID: 24820695. DOI: 10.1007/s10120-014-0381-z
12 Griffin OM, Duggan SN, Ryan R, McDermott R, Geoghegan J and Conlon KC: Characterising the impact of body composition change during neoadjuvant chemotherapy for pancreatic cancer. Pancreatology 19: 850-857, 2019. PMID: 31362865. DOI: 10.1016/j.pan.2019.07.039

13 Huang X, Ma J, Li L and Zhu X: Severe muscle loss during radical chemoradiotherapy for non-metastatic nasopharyngeal carcinoma predicts poor survival. Cancer Med, 2019. PMID: 31517443. DOI: $10.1002 / \mathrm{cam} 4.2538$

14 Lee J, Chang CL, Lin JB, Wu MH, Sun FJ, Jan YT, Hsu SM and Chen YJ: Skeletal muscle loss is an imaging biomarker of outcome after definitive chemoradiotherapy for locally advanced cervical cancer. Clin Cancer Res 24: 5028-5036, 2018. PMID: 29959140. DOI: 10.1158/1078-0432.CCR-18-0788

15 Wells G, Shea B, O'connell D, Peterson J, Welch V, Losos M and Tugwell P: The Newcastle-Ottawa Scale (NOS) for assessing the quality of non-randomised studies in metaanalyses. Ottawa: Ottawa Hospital Research Institute, 2014.

16 Higgins JP and Green S: Cochrane handbook for systematic reviews of interventions, John Wiley \& Sons, 2011.

17 Melnyk B and Fineout-Overholt E: Box 1.3: Rating system for the hierarchy of evidence for intervention/treatment questions. Evidence-based practice in nursing \& healthcare: A guide to best practice. 3rd ed. Philadelphia: Wolters Kluwer Health, 2015.

18 Collins J, Noble S, Chester J, Coles B and Byrne A: The assessment and impact of sarcopenia in lung cancer: a systematic literature review. BMJ Open 4: e003697-2013-003697, 2014. PMID: 24384894. DOI: 10.1136/bmjopen-2013-003697

19 Pamoukdjian F, Bouillet T, Lévy V, Soussan M, Zelek L and Paillaud E: Prevalence and predictive value of pre-therapeutic sarcopenia in cancer patients: a systematic review. Clin Nutr 37: 1101-1113, 2018. PMID: 28734552. DOI: 10.1016/j.clnu.2017.07.010

20 Shachar SS, Williams GR, Muss HB and Nishijima TF: Prognostic value of sarcopenia in adults with solid tumours: a meta-analysis and systematic review. Eur J Cancer 57: 58-67, 2016. PMID: 26882087. DOI: 10.1016/j.ejca.2015.12.030

21 Yoowannakul S, Tangvoraphonkchai K, Vongsanim S, Mohamed A and Davenport A: Differences in the prevalence of sarcopenia in haemodialysis patients: the effects of gender and ethnicity. $\mathrm{J}$ Hum Nutr Diet 31: 689-696, 2018. PMID: 29611250. DOI: $10.1111 /$ jhn. 12555

22 Silva AM, Shen W, Heo M, Gallagher D, Wang Z, Sardinha LB and Heymsfield SB: Ethnicity-related skeletal muscle differences across the lifespan. Am J Hum Biol 22: 76-82, 2010. PMID: 19533617. DOI: $10.1002 /$ ajhb.20956

23 Yoowannakul S, Tangvoraphonkchai K and Davenport A: The prevalence of muscle wasting (sarcopenia) in peritoneal dialysis patients varies with ethnicity due to differences in muscle mass measured by bioimpedance. Eur J Clin Nutr 72: 381, 2018. PMID: 29158495. DOI: 10.1038/s41430-017-0033-6

24 Castaneda C and Janssen I: Ethnic comparisons of sarcopenia and obesity in diabetes. Ethn Dis 15: 664-670, 2005. PMID: 16259491.

25 Stene GB, Helbostad JL, Amundsen T, Sørhaug S, Hjelde H, Kaasa S and Grønberg BH: Changes in skeletal muscle mass during palliative chemotherapy in patients with advanced lung cancer. Acta Oncol 54: 340-348, 2015. PMID: 25225010. DOI: 10.3109/0284186X.2014.953259

26 Kuwada K, Kuroda S, Kikuchi S, Yoshida R, Nishizaki M, Kagawa $\mathrm{S}$ and Fujiwara T: Clinical impact of sarcopenia on 
gastric cancer. Anticancer Res 39: 2241-2249, 2019. PMID: 31092415. DOI: 10.21873/anticanres.13340

27 Reilly CM, Bruner DW, Mitchell SA, Minasian LM, Basch E, Dueck AC, Cella D and Reeve BB: A literature synthesis of symptom prevalence and severity in persons receiving active cancer treatment. Support Care Cancer 21: 1525-1550, 2013. PMID: 23314601. DOI: 10.1007/s00520-012-1688-0

28 Weis J: Cancer-related fatigue: prevalence, assessment and treatment strategies. Expert Rev Pharm Out 11: 441-446, 2011. PMID: 21831025. DOI: 10.1586/erp.11.44

29 Berger AM, Mooney K, Alvarez-Perez A, Breitbart WS, Carpenter KM, Cella D, Cleeland C, Dotan E, Eisenberger MA and Escalante CP: Cancer-related fatigue, version 2.2015. J Natl Compr Canc Netw 13: 1012-1039, 2015. PMID: 26285247. DOI: $10.6004 /$ jnccn.2015.0122

30 Guinan EM, Doyle S, Bennett A, O'Neill L, Gannon J, Elliott J, O'Sullivan J, Reynolds J and Hussey J: Sarcopenia during neoadjuvant therapy for oesophageal cancer: characterising the impact on muscle strength and physical performance. Support Care Cancer 26: 1569-1576, 2018. PMID: 29197960. DOI: 10.1007/s00520-017-3993-0

31 Huang $\mathrm{CH}$, Lue KH, Hsieh TC, Liu SH, Wang TF and Peng TC: Association between sarcopenia and clinical outcomes in patients with esophageal cancer under neoadjuvant therapy. Anticancer Res 40: 1175-1181, 2020. PMID: 32014971. DOI: 10.21873/anticanres. 14060

32 Zhang G, Li X, Sui C, Zhao H, Zhao J, Hou Y and Du Y: Incidence and risk factor analysis for sarcopenia in patients with cancer. Oncol Lett 11: 1230-1234, 2016. PMID: 26893724. DOI: $10.3892 / \mathrm{ol} .2015 .4019$

33 Batsis JA and Villareal DT: Sarcopenic obesity in older adults: aetiology, epidemiology and treatment strategies. Nat Rev Endocrinol 14: 513-537, 2018. PMID: 30065268. DOI: 10.1038/s41574-018-0062-9

34 Hildreth KL, Barry DW, Moreau KL, Vande Griend J, Meacham RB, Nakamura T, Wolfe P, Kohrt WM, Ruscin JM and Kittelson $\mathrm{J}$ : Effects of testosterone and progressive resistance exercise in healthy, highly functioning older men with low-normal testosterone levels. J Clin Endocrinol Metab 98: 1891-1900, 2013. PMID: 23533227. DOI: 10.1210/jc.2012-3695

35 Cho KM, Park H, Oh DY, Kim TY, Lee KH, Han SW, Im SA, Kim TY and Bang YJ: Skeletal muscle depletion predicts survival of patients with advanced biliary tract cancer undergoing palliative chemotherapy. Oncotarget 8: 79441-79452, 2017. PMID: 29108323. DOI: 10.18632/oncotarget.18345

36 Jung AR, Roh J, Kim JS, Kim S, Choi S, Nam SY and Kim SY: Prognostic value of body composition on recurrence and survival of advanced-stage head and neck cancer. Eur J Cancer 116: 98106, 2019. PMID: 31185387. DOI: 10.1016/j.ejca.2019.05.006
37 Kakinuma K, Tsuruoka H, Morikawa K, Furuya N, Inoue T, Miyazawa T and Mineshita M: Differences in skeletal muscle loss caused by cytotoxic chemotherapy and molecular targeted therapy in patients with advanced non-small cell lung cancer. Thorac Cancer 9: 99-104, 2018. PMID: 29067769. DOI: 10.1111/1759-7714.12545

38 Kimura M, Naito T, Kenmotsu H, Taira T, Wakuda K, Oyakawa T, Hisamatsu Y, Tokito T, Imai H and Akamatsu H: Prognostic impact of cancer cachexia in patients with advanced non-small cell lung cancer. Support Care Cancer 23: 1699-1708, 2015. PMID: 25430482. DOI: 10.1007/s00520-014-2534-3

39 Lee J, Lin J, Wu M, Jan Y, Chang C, Huang C, Sun F and Chen $\mathrm{Y}$ : Muscle radiodensity loss during cancer therapy is predictive for poor survival in advanced endometrial cancer. J Cachexia Sarcopenia Muscle, 2019. PMID: 31094101. DOI: 10.1002/jcsm. 12440

40 Li Y, Wang W, Jiang H, Dai J, Xia L, Chen J, Xie C, Peng J, Liao Z and Gao Y: Predictive value of pancreatic dose-volume metrics on sarcopenia rate in gastric cancer patients treated with adjuvant chemoradiotherapy. Clin Nutr 38: 1713-1720, 2019. PMID: 30122263. DOI: 10.1016/j.clnu.2018.07.035

41 Naito T, Okayama T, Aoyama T, Ohashi T, Masuda Y, Kimura M, Shiozaki H, Murakami H, Kenmotsu H and Taira T: Skeletal muscle depletion during chemotherapy has a large impact on physical function in elderly Japanese patients with advanced non-small-cell lung cancer. BMC Cancer 17: 571, 2017. PMID: 28841858. DOI: $10.1186 / \mathrm{s} 12885-017-3562-4$

42 Okuno M, Goumard C, Kopetz S, Vega EA, Joechle K, Mizuno T, Tzeng CD, Chun YS, Lee JE and Vauthey J: Loss of muscle mass during preoperative chemotherapy as a prognosticator for poor survival in patients with colorectal liver metastases. Surgery 165 : 329-336, 2019. PMID: 30197278. DOI: 10.1016/j.surg.2018.07.031

43 Reisinger KW, Bosmans JW, Uittenbogaart M, Alsoumali A, Poeze M, Sosef MN and Derikx JP: Loss of skeletal muscle mass during neoadjuvant chemoradiotherapy predicts postoperative mortality in esophageal cancer surgery. Ann Surg Oncol 22: 44454452, 2015. PMID: 25893413. DOI: 10.1245/s 10434-015-4558-4

44 Rollins KE, Tewari N, Ackner A, Awwad A, Madhusudan S, Macdonald IA, Fearon KC and Lobo DN: The impact of sarcopenia and myosteatosis on outcomes of unresectable pancreatic cancer or distal cholangiocarcinoma. Clin Nutr 35: 1103-1109, 2016. PMID: 26411749. DOI: $10.1016 /$ j.clnu.2015.08.005

Received March 17, 2020

Revised March 23, 2020

Accepted March 24, 2020 